\title{
THE GAUSS MAP AND NONHOLOMORPHIC HARMONIC MAPS
}

\author{
TORU ISHIHARA
}

\begin{abstract}
J. Eells and J. C. Wood [2] gave the fundamental method of construction of certain harmonic maps from Riemann surfaces to complex projective spaces. In this paper, we will construct nonholomorphic harmonic maps of Kaehler manifolds to complex Grassmann manifolds as the Gauss maps for holomorphic immersions of Kaehler manifolds to complex projective spaces.
\end{abstract}

1. Introduction. Let $\mathbf{P}^{N}$ be a complex projective $N$-space. Let $L \rightarrow \mathbf{P}^{N}$ be the canonical line bundle over $\mathbf{P}^{N}$. Let $\underline{\mathbf{C}}^{N+1}$ denote the trivial bundle over $\mathbf{P}^{N}$ with fibre $\mathbf{C}^{N+1}$. Then the holomorphic tangent bundle $T^{\prime} \mathbf{P}^{N}$ of $\mathbf{P}^{N}$ is isomorphic to $L^{*} \otimes L^{\perp}$, where $L^{*}$ is the dual of $L$ and $L^{\perp}$ is the orthogonal complement of $L$ in $\underline{\mathbf{C}}^{N+1}$. Using this isomorphism, for any point $y$ of $\mathbf{P}^{N}$, we can regard a linear subspace of $T_{y}^{\prime} \mathbf{P}^{N}$ as a linear subspace of $\mathbf{C}^{N+1}$. Let $f: M \rightarrow \mathbf{P}^{N}$ be a holomorphic immersion of a Kaehler $n$-space $M$ to $\mathbf{P}^{N}$. Since $d f\left(T_{x}^{\prime} M\right), x \in M$, is viewed as a complex $n$-subspace of $\mathbf{C}^{N+1}$, we can define the Gauss map $g$ of $M$ to a flag manifold $F_{n, n+1}=U(N+1) / U(n) \times U(N-n) \times U(1)$ by $g(x)=$ $\left(d f\left(T^{\prime} M\right),\{f(x)\}\right)$, where $\{f(x)\}$ is the complex line determined by $f(x)$. Let $\pi_{1}$ (resp. $\pi_{2}$ ) be the projection of $F_{n, n+1}$ to $F_{n}=U(N+1) / U(n) \times U(N-n+1)$ (resp. $\left.F_{n+1}=U(N+1) / U(n+1) \times U(N-n)\right)$. Put $g_{i}=\pi_{i} g(i=1,2)$, these will also be called Gauss maps. Let $V$ be a simply connected complete $N$-space of constant holomorphic curvature. Nishikawa [6] defined the Gauss map for a holomorphic immersion of a Kaehler manifold into $V$. In the case $V=$ a complex projective space, the Gauss map of his sense is the same as the map $g_{2}$ in our sense. He showed that his Gauss map is antiholomorphic (-holomorphic) [6]. We show that if an immersion is not totally geodesic, the corresponding maps $g$ and $g_{1}$ are not \pm holomorphic (Proposition 1). But they are always harmonic (Theorem 5). Hence for every holomorphic immersion $f: M \rightarrow \mathbf{P}^{N}$ which is not totally geodesic, we can construct harmonic mappings $g: M \rightarrow F_{n, n+1}$ and $g_{1}: M \rightarrow F_{n}$ which are not \pm holomorphic. Let $M$ be a Riemann surface. Then it is easy to show that our map $\bar{g}_{1}: M \rightarrow F_{n}$ is the same as Eells-Wood's map $\phi$ produced in [2, Proposition 3.10 (with $r=1$ )]. Recently S. Erdem and J. C. Wood [3] constructed all isotropic harmonic maps from a Riemann surface into a complex Grassmann manifold.

Received by the editors May 13, 1982 and, in revised form, March 17, 1983. 1980 Mathematics Subject Classification. Primary 53C40, 53C55.

Key words and phrases. Gauss map, harmonic map, holomorphic immersion. 
2. Kaehler immersions. It will be agreed that our indices have the following ranges throughout this paper; $0 \leqslant A, B, C \leqslant N, 1 \leqslant a, b, c \leqslant N, 1 \leqslant i, j, k \leqslant n, n+1 \leqslant$ $r, s, t \leqslant N, 0 \leqslant i^{*}, j^{*}, k^{*} \leqslant n, n+1 \leqslant r^{*}, s^{*}, t^{*} \leqslant N$ or $r^{*}, s^{*}, t^{*}=0$.

Let $F \mathbf{P}^{N}$ be the bundle of unitary frames of $\mathbf{P}^{N}$. Let $F^{*} \mathbf{P}^{N}$ be the set of elements $b=\left(y, e_{0}, e_{1}, \ldots, e_{N}\right)$ such that $e_{0} \in C^{N+1},\left\|e_{0}\right\|=1, d \pi\left(e_{0}\right)=0$ and $\left(y, e_{1}, \ldots, e_{N}\right) \in F \mathbf{P}^{N}$, where $\pi: C^{N+1} \rightarrow \mathbf{P}^{N}$ is the natural projection. Then $F^{*} \mathbf{P}^{N}$ is a principal bundle over $\mathbf{P}^{N}$ with group $U(1) \times U(N)$. Since $T_{y}^{\prime} \mathbf{P}^{N} \simeq L_{y}^{*} \times L_{y}^{\perp}$, each $e_{a}$ is regarded as a linear map $e_{a}: L_{y} \rightarrow L_{y}^{\perp}$. Moreover, by identifying $e_{a}$ with $e_{a}\left(e_{0}\right)$, we view $F^{*} \mathbf{P}^{N}$ as a subspace of $\mathbf{P}^{N} \times U(N+1)$.

Let $\phi_{B}^{A}$ be the Maurer-Cartan forms of the unitary group $U(N+1)$. Then $\phi_{B}^{A}+\bar{\phi}_{A}^{B}=0$ and $d \phi_{B}^{A}=\Sigma \phi_{B}^{C} \wedge \phi_{C}^{A}$. Let $\rho: \mathbf{P}^{N} \times U(N+1) \rightarrow U(N+1)$ be the natural projection. Denote by $\theta^{a}$ (resp. $\left.\theta_{b}^{a}\right)$ the restriction of $\rho^{*} \phi_{0}^{a}\left(\right.$ resp. $\left.\rho^{*} \phi_{b}^{a}-\delta_{b}^{a} \rho^{*} \phi_{0}^{0}\right)$ to $F^{*} \mathbf{P}^{N}$. Then the Kaehler metric on $\mathbf{P}^{N}$ is given locally by $d \sigma^{2}=2 \Sigma \theta^{a} \bar{\theta}^{a}$ and the structure equations in $\mathbf{P}^{N}$ are written as (cf. [6])

$$
\left\{\begin{array}{l}
d \theta^{a}=\Sigma \theta^{b} \wedge \theta_{b}^{a}, \\
d \theta_{b}^{a}=\Sigma \theta_{b}^{c} \wedge \theta_{c}^{a}+\theta^{a} \wedge \bar{\theta}^{b}+\delta_{b}^{a} \Sigma \theta^{c} \wedge \bar{\theta}^{c} .
\end{array}\right.
$$

Let $M$ be a Kaehler $n$-manifold isometrically immersed into $\mathbf{P}^{N}$ by a holomorphic mapping $f: M \rightarrow \mathbf{P}^{N}$. Let $F M$ be the bundle of unitary frames on $M$. Let $F^{*} M$ denote the set of elements $b=\left(x, e_{0}, \ldots, e_{N}\right)$ such that $\left(x, e_{1}, \ldots, e_{n}\right) \in F M$ and $\left(f(x), e_{0}, d f\left(e_{1}\right), \ldots, d f\left(e_{n}\right), e_{n+1}, \ldots, e_{N}\right) \in F^{*} \mathbf{P}^{N}$. Then $F^{*} M$ is a principal bundle over $M$ with group $U(n) \times U(N-n) \times U(1)$. The natural immersion $\tilde{f}: F^{*} M \rightarrow$ $F^{*} \mathbf{P}^{N}$ is defined by $\tilde{f}(b)=\left(f(x), e_{0}, d f\left(e_{1}\right), \ldots, d f\left(e_{n}\right), e_{n+1}, \ldots, e_{N}\right) \in F^{*}\left(\mathbf{P}^{N}\right)$. On putting $\omega^{a}=\tilde{f}^{*} \theta^{a}$ and $\theta_{b}^{a}=\tilde{f}^{*} \theta_{b}^{a}$, we have $\omega^{r}=0$ and we can put $\omega_{k}^{r}=\sum A_{k j}^{r} \omega^{k}$, where $A_{k j}^{r}$ are regarded as the components of the second fundamental form of $f: M \rightarrow \mathbf{P}^{N}$. The Kaehler metric on $M$ is given locally by $d s^{2}=2 \Sigma \theta^{i} \overline{\boldsymbol{\theta}}^{i}$. From (1), we obtain the following structure equations in $M$

$$
d \omega^{i}=\Sigma \omega^{k} \wedge \omega_{k}^{i}, \quad d \omega_{j}^{i}=\Sigma \omega_{j}^{k} \wedge \omega_{k}^{i}+\Sigma\left(\delta_{i k} \delta_{j l}+\delta_{i j} \delta_{k l}-\sum A_{j k}^{r} \bar{A}_{i l}^{r}\right) \omega^{k} \wedge \bar{\omega}^{l} .
$$

Let $-2 i \sum R_{l} \omega^{l} \wedge \bar{\omega}^{k}$ be the Ricci form of $M$ (in this paper, $\bar{i}, \bar{j}, \bar{k}$ run from $\bar{l}$ to $\bar{n}$ ). Then it holds

$$
\sum A_{i j}^{r} \bar{A}_{i k}^{r}=(n+1) \delta_{j k}-R_{j k} .
$$

3. The Gauss map. Let $\tilde{g}$ be the composition of the natural immersion

$$
\tilde{f}: F^{*} M \rightarrow F^{*} \mathbf{P}^{N} \subset \mathbf{P}^{N} \times U(N+1)
$$

and the natural projection

$$
\rho: \mathbf{P}^{N} \times U(N+1) \rightarrow U(N+1) .
$$

Then $\tilde{g}$ is viewed as a bundle map

$$
\begin{array}{ccc}
F^{*} M & \stackrel{\tilde{g}}{\rightarrow} & U(N+1) \\
\downarrow & & \downarrow \\
M & \rightarrow & F_{n, n+1}
\end{array}
$$


The corresponding base map $g$ is just the Gauss map given in $\S 1$. Let $g_{1}$ and $g_{2}$ also be as in $\$ 1$.

Examples. (1) Let $f: \mathbf{P}^{N} \rightarrow \mathbf{P}^{N}$ be an imbedding of $\mathbf{P}^{n}$ as a projective subspace of $\mathbf{P}^{N}$. Let $f^{\prime}$ be the corresponding map of the set of linear subspaces of $\mathbf{C}^{n+1}$ to the set of linear subspaces of $\mathbf{C}^{N+1}$. Then $g_{2}$ is a constant map $g_{2}(x)=f^{\prime}\left(\mathbf{C}^{n+1}\right) \in F_{n+1}$, $x \in \mathbf{P}^{n}$. For $x \in \mathbf{P}^{n}$, let $x$ be the polar space of $x$. Then the map $f^{\prime}$ defines the canonical imbedding $f^{\prime \prime}$ of a Grassmann manifold $G(n, 1)$ to $F_{n}=G(n, N-n+1)$. Then $g_{1}(x)=f^{\prime \prime}(x) \in F_{n}, x \in \mathbf{P}^{n}$.

(2) Let $f: Q_{N-1} \rightarrow \mathbf{P}^{N}$ be the canonical imbedding of the hyperquadric in $\mathbf{P}^{N}$. Let $\bar{x}$ be the complex conjugate line of a complex line $x \in \mathbf{P}^{N}$. Then $g_{1}(x)=(x \cup \bar{x})^{\perp}$ $\in F_{N-1}$ and $g_{2}(x)=\bar{x}^{\perp} \in F_{N}$.

We introduce a quadratic differential form $d \Sigma^{2}=2 \Sigma \phi_{0}^{i} \bar{\phi}_{0}^{i}+2 \Sigma \phi_{r}^{i *} \bar{\phi}_{r}^{i *}$ and a complex structure $J$ on the flag manifold $F_{n, n+1}$ by $J \phi_{0}^{j}=i \phi_{0}^{j}, J \phi_{r}^{i^{*}}=i \phi_{r}^{i^{*}}$. Then $\left(d \Sigma^{2}, J\right)$ gives a Hermitian structure of $F_{n, n+1}$. From the definition of the Gauss map $g$, we can see

$$
g^{*}\left(\phi_{0}^{i}\right)=\omega^{i}, \quad g^{*}\left(\phi_{r}^{0}\right)=0, \quad g^{*}\left(\phi_{r}^{i}\right)=-\Sigma \bar{A}_{i j}^{r} \bar{\omega}^{j} .
$$

A Kaehler metric $d \Sigma_{1}^{2}$ (resp. $d \Sigma_{2}^{2}$ ) on $F_{n}$ (resp. $F_{n+1}$ ) is given by $d \Sigma_{1}^{2}=2 \sum \phi_{r^{*}}^{i} \bar{\phi}_{r^{*}}^{i}$ (resp. $d \Sigma_{2}^{2}=2 \sum \phi_{r}^{i^{*} \bar{\phi}_{r}^{i *}}$ ) and a complex structure $J_{1}$ (resp. $J_{2}$ ) on $F_{n}$ (resp. $F_{n+1}$ ) is given by $j_{1} \phi_{r^{*}}^{i}=i \phi_{r^{*}}^{i}\left(\right.$ resp. $\left.J_{2} \phi_{r}^{i^{*}}=i \phi_{r}^{i^{*}}\right)$. We have also

$$
\begin{array}{ll}
g_{1}^{*}\left(\phi_{0}^{i}\right)=\omega^{i}, & g_{1}^{*}\left(\phi_{r}^{i}\right)=-\sum \bar{A}_{i j}^{r} \bar{\omega}^{j}, \\
g_{2}^{*}\left(\phi_{r}^{0}\right)=0, & g_{2}^{*}\left(\phi_{r}^{i}\right)=-\sum \bar{A}_{i j}^{r} \bar{\omega}^{j} .
\end{array}
$$

Using (2), we can show

(6) $g^{*}\left(d \Sigma^{2}\right)=g_{1}^{*}\left(d \Sigma_{1}^{2}\right)=d s^{2}+\sum A_{i j}^{r} \bar{A}_{i k}^{r} \omega^{j} \bar{\omega}^{k}=(n+2) d s^{2}-\sum R_{j k} \omega^{k} \bar{\omega}^{k}$,

(7) $g_{2}\left(d \Sigma_{2}^{2}\right)=\sum A_{i j}^{r} \bar{A}_{i k}^{r} \omega^{j} \bar{\omega}^{k}=(n+1) d s^{2}-\sum R_{j k} \omega^{j} \bar{\omega}^{k}$.

Nishikawa [6] proved that $g_{2}$ is antiholomorphic and that $g_{2}$ is a constant map iff the immersion $f$ is totally geodesic. From (6) it follows

PROPOSITION 1. The immersion $f$ is totally geodesic iff $g$ is isometric and holomorphic, and this holds iff $g_{1}$ is isometric and holomorphic.

As the complex version of some Obata's results in [5], we get

Proposition 2. Let $f: M \rightarrow \mathbf{P}^{N}$ be a holomorphic and isometric immersion. The following are equivalent: (1) $M$ Einstein; (2) $g$ a homothety or constant map; (3) $g_{1} a$ homothety or constant map; (4) $g_{2}$ a homothety or constant map.

4. The harmonic Gauss map. Put

$$
\phi_{j^{*} s^{*}}^{i * r^{*}}=\phi_{j^{*}}^{i^{*}} \delta_{s^{*}}^{r^{*}}-\delta_{j^{*}}^{i^{*} \phi_{r^{*}}^{s^{*}} .}
$$

Then the structure equations in the Kaehler manifold $F_{n}$ (resp. $F_{n+1}$ ) are given by

$$
d \phi_{r^{*}}^{i}=-\sum \phi_{j s^{*}}^{i r^{*}} \wedge \phi_{s^{*}}^{j} \quad\left(\text { resp. } d \phi_{r}^{i^{*}}=-\Sigma \phi_{j^{*} s}^{i^{*} r} \wedge \phi_{s}^{j^{*}}\right) .
$$


On the other hand, the structure equations in $F_{n, n+1}$ are given by

$$
\begin{aligned}
& d \phi_{0}^{i}=-\sum \phi_{j r^{*}}^{i 0} \wedge \phi_{r^{*}}^{j}, \\
& d \phi_{r}^{0}=-\sum \phi_{j^{*} r}^{0 r} \wedge \phi_{r}^{j^{*}}, \\
& d \phi_{r}^{i}=-\sum \phi_{j s^{*}}^{i r} \wedge \phi_{s^{*}}^{j}-\sum \phi_{0 s}^{i r} \wedge \phi_{s}^{0}+\phi_{0}^{i} \wedge \phi_{r}^{0} .
\end{aligned}
$$

Hence the Hermitian structure $\left(d \Sigma^{2}, J\right)$ is not Kaehlerian. Let $B_{j}^{i 0}, B_{j}^{i 0}, B_{j}^{0 r}, B_{j}^{0 r}$, $B_{j}^{i r}, B_{j}^{i r}$ be the coefficients of the Jacobian matrix $J(g)$, that is, $g^{*}\left(\phi_{0}^{i}\right)=\sum B_{j}^{i 0} \omega^{j}+$ $\sum B_{j}^{i 0} \bar{\omega}^{j}$ and so on. Then from (3), it follows that $B_{j}^{i 0}=\delta_{j}^{i}, B_{j}^{i r}=-\bar{A}_{i j}^{r}$ and any other components vanish. We will calculate the covariant derivative of the Jacobian $J(g)$. We can put [1]

$$
\begin{aligned}
\sum B_{j k}^{i 0} \omega^{k}+\sum B_{j k}^{i 0} \bar{\omega}^{k}= & d B_{j}^{i 0}-\sum B_{k}^{i 0} \omega_{j}^{k}+\sum B_{j}^{k 0} g^{*}\left(\phi_{k 0}^{i 0}\right) \\
& +\sum B_{j}^{0 r} g^{*}\left(\phi_{0 r}^{i 0}\right)+\sum B_{j}^{k r} g^{*}\left(\phi_{k r}^{i 0}\right)=0 .
\end{aligned}
$$

Hence $B_{j k}^{i 0}=B_{j k}^{i 0}=0$. Similarly we can show that all $B_{j k}^{i 0}, B_{j k}^{i 0}, B_{j k}^{0 r}, B_{j k}^{0 r}, B_{j k}^{0 r}, B_{j k}^{i r}, B_{j k}^{i r}$ vanish. By exterior differentiation of the last equation in (3), we get

$$
\left(\sum B_{j k}^{i r} \omega^{k}+\sum B_{j k}^{i r} \bar{\omega}^{k}\right) \wedge \bar{\omega}^{j}=0 .
$$

Thus we obtain

LEMMA 3. The only nonvanishing components of the covariant derivative of the components of the Jacobian matrix $J(g)$ are $B_{j k}^{i r}$.

Similarly we put $g_{1}^{*}\left(\phi_{r^{*}}^{i}\right)=\Sigma C_{j}^{i r^{*}} \omega^{j}+\Sigma C_{j}^{i r^{*}} \bar{\omega}^{j}$. Then $C_{j}^{i 0}=\delta_{j}^{i}, C_{j}^{i r}=0, C_{j}^{i 0}=0$, $C_{j}^{i r}=-\bar{A}_{i j}^{r}$, and we can prove

LEMMA 4. The only nonvanishing components of the covariant derivative of the components of the Jacobian matrix $J\left(g_{1}\right)$ are $C_{j k}^{i r}$.

From Lemma 3, it follows that $\Sigma B_{j j}^{i 0}=0, \Sigma B_{j j}^{0 r}=0$ and $\Sigma B_{j j}^{i r}=0$. Hence $g$ is harmonic (see [1]). Similarly $g_{1}$ is harmonic. Thus we get

THEOREM 5. Let $f: M \rightarrow \mathbf{P}^{N}$ be a holomorphic and isometric immersion of a Kaehler $n$-manifold $M$. Then the corresponding Gauss maps $g: M \rightarrow F_{n, n+1}$ and $g_{1}: M \rightarrow F_{n}$ are harmonic.

Let $\pi_{1}: F_{n, n+1} \rightarrow F_{n}$ be the natural projection. Then we have $\pi_{1}^{*}\left(\phi_{r^{*}}^{i}\right)=\phi_{r^{*}}^{i}$. Combining this with (3), we get (the definition of $\pi_{1}$-horizontal maps is found in $[2,4]$ ),

Proposition 6. The Gauss map $g$ is $\pi_{1}$-horizontal.

Let $M$ be a compact complex manifold and $L \rightarrow M$ be a holomorphic line bundle. Every linear system of divisors for $L \rightarrow M$ defines a holomorphic mapping of $M$ to $\mathbf{P}^{N}$. In particular, the Kodaira imbedding theorem asserts that if $L \rightarrow M$ is a positive line bundle, there is an imbedding of $M$ to $\mathbf{P}^{N}$. Hence using Theorem 5 , we can construct a lot of nonholomorphic harmonic maps of $M$ to complex Grassmann manifolds. 
ACKNOWLEDGEMENT. This research was mainly carried out while the author was at the University of Warwick. The author is grateful to the University of Warwick for its hospitality.

\section{REFERENCES}

1. S. S. Chern and S. I. Goldberg, On the volume decreasing property of a class of real harmonic mappings, Amer. J. Math. 97 (1975), 133-147.

2. J. Eells and J. C. Wood, Harmonic maps from surfaces to complex projective spaces, Adv. in Math. (to appear).

3. S. Erdem and J. C. Wood, On the construction of harmonic maps into a Grassmannian, London Math. Soc. (to appear)

4. T. Ishihara, The harmonic Gauss maps in a generalized sense, J. London Math. Soc. 26 (1982), 104-112.

5. M. Obata, The Gauss map of immersions of Riemannian manifolds in space of constant curvature, J. Differential Geom. 2 (1968), 217-223.

6. S. Nishikawa, Gauss map of Kaehler immersions, Tôhoku J. Math. 27 (1975), 453-460.

Department of Mathematics, Tokushima University, Tokushima, JaPan 